a fetus with Edwards syndrome. She was asymptomatic post administration of mifepristone 48 hours prior to admission. As an inpatient she was given 5 doses of misoprostol (400 micrograms) vaginally. Lack of progress necessitated 4 further doses after 24 hours break. The patient had a persistent pyrexia of above 38 degrees following $4^{\text {th }}$ dose of misoprostol which was attributed to prostaglandin administration. Investigations revealed a CRP of 226 and a white cell count of 19.1. She was haemodynamically stable and used a remifentanyl PCA for analgesia. In view of lack of response to Misoprostol she was rescanned revealing a bulky uterus with the fetus lying intraperitoneally with an intact amniotic sac. CT scan revealed a $6.3 \mathrm{~cm}$ defect in the anterior uterine wall with an intact sac projecting beyond normal uterine contour. There was no free fluid. In view of this patient had a laparotomy revealing a 6 by $5 \mathrm{~cm}$ defect in the anterior uterine wall lined by necrotic tissue. In view of significant amount of necrosis a decision was made to proceed with subtotal hysterectomy. We wish to highlight this rare case wherein uterine rupture occurred due to infection and uterine wall necrosis possibly secondary to amniocentesis and fetal demise. All the classical features of uterine rupture like haemodynamic instability and intraperitoneal bleeding were absent. Only a high index of suspicion leading on to further imaging helped establish the diagnosis.

\section{PM.78 USE OF INTRAVENOUS IRON SUCROSE INJECTIONS IN THE TREATMENT OF IRON DEFICIENCY ANAEMIA IN PREGNANCY}

doi:10.1136/archdischild-2013-303966.159

'A Butt, ${ }^{2} \mathrm{M}$ Broderick, 'E Ciantar. 'Leeds Teaching Hospitals NHS Trust, Leeds, UK; 'University of Leeds, Leeds, UK

Background Iron deficiency anaemia (IDA) is the commonest cause of anaemia in pregnancy affecting $19 \%$ of the Leeds pregnant population ${ }^{1}$. It is associated with maternal morbidity including fatigue and postpartum depression ${ }^{2}$. It is also linked to adverse pregnancy outcomes including preterm delivery and intrauterine growth restriction. ${ }^{3}$

Aim To assess the use of intravenous iron sucrose antenatally in women with iron deficiency anaemia who were intolerant to oral treatment and its efficacy in increasing haemoglobin levels.

Method There were 23 patients who received iron sucrose injections in the antenatal period, identified retrospectively from the antenatal day records in the Leeds Teaching Hospitals.

Results Out of 23 patients in our cohort, 9 (39\%) were given iron sucrose injections after 37 weeks gestation. 2 women (9\%) received the injections between $20-30$ weeks. The remaining 12 patients (52\%) had their injections between $31-36$ weeks. The mean gestation for commencing treatment was 34 weeks. The mean cohort haemoglobin level before treatment was $8.5 \mathrm{~g} / \mathrm{dl}$ which improved to $9.9 \mathrm{~g} / \mathrm{dl}$ after treatment. The maximum number of doses of iron sucrose injections used was 4 . None of the patients required blood transfusion post-delivery. 2 patients $(9 \%)$ delivered preterm between 31-37 weeks. 20 babies (87\%) were born with an average birth weight between $2.5 \mathrm{~kg}-4.0 \mathrm{~kg}$.

Conclusion Iron sucrose injections are very effective in increasing the haemoglobin level antenatally in women who can't tolerate the oral preparation or very severely anaemic.

\section{REFERENCES}

1. Lee J, Chang C. Audit on Iron Deficiency in Pregnancy in Leeds - Student Selected Component project.

2. Crown EJ et al, Low Hemoglobin Level is a Risk Factor for Postpartum Depression. The Journal of Nutrition 2003 133:4139-42.

3. Williams MD, Wheby MS. Anaemia in pregnancy. Med Clin North Am 1992 $76(3): 631-4$.

\section{PM.79 A REVIEW OF PREGNANT WOMEN ON TACROLIMUS AND PREGNANCY OUTCOMES IN LIVERPOOL}

doi:10.1136/archdischild-2013-303966.160

'S Ballal, ${ }^{2} \mathrm{~N}$ Phelan, ${ }^{2} \mathrm{M}$ Howse, 'S Walkinshaw. 'Liverpool Womens NHS foundation Trust, Liverpool, UK; ${ }^{2}$ Royal Liverpool University Teaching Hospita, Liverpool, UK

Background Fertility is usually restored in women with renal transplants, one series noting pregnancy occurring in $12 \%$ of women at childbearing age. ${ }^{(1)}$

Tacrolimus is the primary immunosuppressant in renal transplant patients. Improved transplant outcome correlates with increasing antenatal population receiving immunosuppressive therapy ${ }^{(2)}$. As pregnancy progresses, hepatic P450 cytochrome may be inhibited which thought to lead to increased serum Tacrolimus levels. We think circulating plasma volume increases and this would drop Tacrolimus levels by a dilutional effect.

Methods Retrospective analysis of notes from 2000-2012 from Renal-Obstetric clinic.

Results 15 pregnancies in 11 women on Tacrolimus recorded. Mean maternal age 30.1. Mean transplantation age 23.9 years. 5 patients had multiple renal transplants.

6 patients were primiparous (40\%) and 9 multiparous $(60 \%)$. Only 5 women received pre-pregnancy counselling.

10 pregnancies required increasing antenatal Tacrolimus dosing. 3 patients developed worsening renal function.

Mean gestation reached 30 weeks, with $80 \%$ of deliveries occurring $<37$ weeks. Live birth rate was $73.3 \%$. 4 patients $(26.7 \%)$ entered spontaneous labour, $5(33.3 \%)$ required induction $(33.3 \%)$ and 6 caesarean section deliveries (40\%). Only 3 patients (20\%) attended post natal follow-up within 6 weeks post-delivery.

Conclusions Our findings suggest dropping Tacrolimus levels in the late first and early second trimester, but then levels appear to gradually increase. Our live birth, IUGR and emergency caesarean rate were in consistence with rates noted in previous studies ${ }^{(3)}$. More research needed looking at the effect of fluctuant Tacrolimus levels and if a drop in first trimester is associated with adverse pregnancy outcome.

\section{REFERENCES}

1. Sturgiss SN, Davison JM. Effect of pregnancy on long-term function of renal allografts. Am J Kidney Dis 1992:167-72.

2. Webster AC, Woodroffe RC, Taylor RS, Chapman JR, Craig JC. Tacrolimus versus ciclosporin as primary immunosuppression for kidney transplant recipients: metaanalysis and meta-regression of randomised trial data. BMJ 2005;331:810

3. Pregnancy in Renal Transplant Recipients: A UK National Cohort Study CJASN CJN. K Bramham, C Nelson-Piercy, H Gao, M Pierce, N Bush, P Spark, P Brocklehurst, J Kurinczuk, M Knight.

\section{PM.80 RETRIEVABLE INFERIOR VENA CAVA (IVC) PHILTRES IN PREGNANCY - A CASE SERIES AND REVIEW OF THE LITERATURE}

doi:10.1136/archdischild-2013-303966.161

P Paramanathan, N Maher, K Ryan, N McEniff, C Lynch, C Regan, B Byrne. Coombe Women \& Infants University Hospital, Dublin, Ireland

We report 3 cases of retrievable IVC philtres in pregnancy and performed a systematic review of the literature to determine the indications, success and complications rates for these devices in pregnancy.

A literature search of PubMed and Medline was conducted using the terms IVC philtre and pregnancy.

In our unit, 2 women had peripartum pulmonary emboli (PE) and the third woman had an iliac vein thrombosis and recurrent antepartum haemorrhage. Insertion of philtres was uncomplicated but only one was retrieved post delivery. 
A total of 48 patients were identified from 20 publications (51 including our patients). Indications for insertion included extensive deep vein thrombosis (DVT) (39), PE (7), recurrent thrombosis (1), contraindication to anticoagulation (1) and unknown (3). Complications at insertion occurred in 2 patients. Two philtres were not removed, one philtre was repositioned and outcome was unclear in 5 . Therefore $37 / 40$ were retrieved successfully (81\%). Retrieval failed in 8 women because of failure to snare (2), tilting (4), fracture and migration of the device(1) and occlusion of the philtre with thrombus (1).

The incidence of failed retrieval of IVC philtres in pregnancy $(18 \%)$ is at the higher end of the spectrum reported in the general population $(0-22 \%)$. Careful consideration of the benefits and risks of IVC philtre placement in pregnancy is paramount and the patient needs to be informed that almost one in five philtres remain in situ with subsequent increased risk of post thrombotic syndrome.

\section{PM.81 THE IMPACT OF AN OBSTETRIC ANAESTHETIC ANTENATAL CLINIC FOR THE MORBIDLY OBESE, A RETROSPECTIVE STUDY}

doi:10.1136/archdischild-2013-303966.162

A Patience, MWJ MacDougall. Royal Victoria Infirmary, Newcastle Upon Tyne, UK

The CMACE/RCOG joint guideline recommend in their management of women with obesity in pregnancy guideline that pregnant women with a booking BMI > 40 should have an antenatal consultation with an obstetric anaesthetist. We compare the pregnancy outcome data in our morbidly obese population from both before and after the introduction of our specialist obesity obstetric anaesthetic clinic.

We performed a retrospective audit, comparing pregnancy outcomes in the first six months of 2009 pre-clinic, and the first six months of 2011 post clinic establishment, in the morbidly obese, looking at; anaesthetic type, estimated blood loss, cord $\mathrm{pH}<7.1$, cord $\mathrm{pH}<7.0$, and APGARs at 1, 5 and 10 minutes. The results are reviewed in the context of mode of delivery, induction, preterm delivery and birth weight.

Results The type of anaesthetic for elective section was 12/12 $(100 \%)$ spinal in 2009 v $10 / 11(90.9 \%)$ spinal $+1 / 11(9.1 \%)$ epidural in 2011. For emergency section the type of anaesthetic was $3 / 12$ $(25 \%)$ epidural $+9 / 12(75 \%)$ spinal in 2009 v $10 / 15(66.7 \%)$ epidural with one conversion to general anaesthetic $(6.7 \%)+5 / 15(33.3 \%)$ spinal in 2011. Estimated blood loss in 2011 appeared higher than in 2009 , but was not significantly so. Comparing EBL in 2009 v 2011; $23 / 46(50 \%)$ v35/70 (50\%) had $<500 \mathrm{ml}, 20 / 46$ (43.3\%) v25/70 $(35.7 \%)$ had $500-999 \mathrm{ml}, 1 / 46(2.1 \%)$ v $4 / 70$ (5.7\%) lost $1000-$ $1499 \mathrm{ml}, 2 / 46(4.3 \%)$ v 5/70 (7.1\%) lost $>1500 \mathrm{ml}$. Cord $\mathrm{pH}$ below 7.0 occurred in $1 / 46(2.1 \%)$ in 2009 v $1 / 70(1.4 \%)$ in 2011 . APGARS below 8 at 1,5 and 10 minutes were $3 / 46(6.5 \%), 1 / 46(2.1 \%)$ and $1 / 46(2.1 \%)$ respectively in 2009 and $13 / 70$ (18.6\%), 2/70 (2.8\%) and $0 / 70(0 \%)$ respectively in 2011 .

There were no obvious improvements in our outcome measures following the introduction of a consultant obstetric anaesthetist clinic for those with morbid obesity.

\section{PM.82 ACUTE HYPONATRAEMIA IN LABOUR - THE OBSTETRIC MARATHON?}

doi:10.1136/archdischild-2013-303966.163

P Sokhal, J Cook, S Robinson, L Lakasing. St Mary's Hospital, Imperial College Healthcare, London, UK

Maternal hyponatraemia during labour can affect both mother and baby. As a result, standard intrapartum care includes administration of oxytocin in sodium-containing fluids, limitation of oral intake and use of isotonic sports drinks. There is no strict guidance on best practise and local protocols vary.

We present a case report of acute severe hyponatraemia following spontaneous vaginal delivery at $38^{+5}$ weeks gestation in a 34-year-old primiparous woman. This previously well woman spent 4.5 hours in the birthing pool and drank approximately 6 litres of water/lucozade in that time. After delivery, she suffered a seizure and acute confusion. The plasma sodium was $117 \mathrm{mmol} / \mathrm{L}$ (135$145 \mathrm{mmol} / \mathrm{l})$. This was corrected with hypertonic saline to 130 $\mathrm{mol} / \mathrm{L}$ within 4 hours. She was admitted to intensive care and required sedation and ventilation. Endocrine investigations revealed no underlying cause. Differential diagnoses included atypical eclampsia and posterior-reversible encephalopathy syndrome. She was discharged on day 6 with a Mini-mental State Examination score of $30 / 30$

Isotonic drinks prevent urinary ketosis, maintain plasma glucose and electrolytes, thereby preventing the 'starvation effect' of labour, also seen in marathon runners. Review of the literature relating to the effect of water immersion and oral fluid intake on plasma sodium levels during labour suggests that a 40-minute bath may cause increased naturiesis and plasma volume expansion. Water tolerance appears diminished in labour and thus intoxication may be possible with relatively moderate volumes.

We recommend that in labour a) women do not drink excessively, b) hypotonic fluid administration is avoided, c) fluid-balance charts become mandatory.

\section{PM.83 TESTING FOR FETOMATERNAL HAEMORRHAGE BY ACID ELUTION CAN YIELD FALSE POSITIVE RESULTS IN THE PRESENCE OF ELEVATED MATERNAL FETAL HAEMOGLOBIN}

doi:10.1136/archdischild-2013-303966.164

A Doyle, J Donnelly, S Campbell, D Murphy, D Corcoran, B Kumpel, FD Ni Ainle. Rotunda Hospital, Rotunda, Ireland

Appropriate testing for fetomaternal haemorrhage (FMH) is critical in the prevention of morbidity and mortality due to haemolytic disease of the fetus and newborn (HDFN) in $\mathrm{RhD}$ negative women. The Kleihauer or acid elution (AE) test is widely used to assess the size of fetomaternal haemorrhage and to determine whether sufficient Anti-D immunoglobulin has been administered to prevent HDFN. This test is based on the principle that adult haemoglobin $(\mathrm{HbA})$ is eluted from red cells in an acidic solution while the fetal haemoglobin $(\mathrm{HbF})$ is not. However, in the presence of an elevated level of $\mathrm{HbF}$ of maternal origin, the AE test may be "positive" in the absence of a true FMH. We report two cases in which this situation arose antenatally, leading to difficulties in clinical interpretation. In both cases, specialised flow cytometry revealed the presence of elevated levels of $\mathrm{HbF}$ of maternal origin. While awaiting completion of specialised investigations, Anti-D was administered but was later found to have been unnecessary. Early awareness of the possibility of elevated maternal F cells ensures that samples can be sent to appropriate reference laboratories early to limit unnecessary Anti-D administration. The true prevalence of "false positive" AE tests due to elevated maternal $\mathrm{HbF}$ is unknown and is the subject of ongoing work in our laboratory, but should be considered in the differential diagnosis of an $\mathrm{AE}$ test remaining positive despite appropriate Anti-D administration.

\section{PM.84 THE ASSOCIATION BETWEEN SEVERITY OF IMPAIRED GLUCOSE TOLERANCE IN GESTATIONAL DIABETES WITH AGE, BMI AND ETHNICITY}

doi:10.1136/archdischild-2013-303966.165

B Jones, S Balaji, C Cotzias. The West Middlesex University Hospital, Isleworth, UK 